10.21611/qirt.2019.025

\title{
Virtual Wave Concept for 3D Thermographic Imaging
}

\author{
by G. Mayr*, G. Thummerer*, H. Plasser*, P. Burgholzer** \\ * University of Applied Sciences Upper Austria, Josef Ressel Centre for Thermal NDE of Composites Franz- \\ Fritsch-Straße 11, 4600, Wels, Austria, holger.plasser@fh-wels.at \\ ${ }^{* *}$ RECENDT - Research Center for Non Destructive Testing GmbH, Altenberger Straße 69, 4040, Linz, Austria, \\ peter.burgholzer@recendt.at
}

\begin{abstract}
In this work, the Virtual Wave Concept is applied for a 3D reconstruction of the internal structure using the measured surface temperature. Thermal waves are generated with a heat source, transmitted into the solid and received by a measurement with an IR camera. Because of traversing and interacting with the specimen, the thermal wave carries information about the inner structure. The thermal waves are converted by a local transformation into virtual waves. The propagation of the virtual waves obeys the wave equation, whereby the information of the inner structure can be extracted with ultrasonic image reconstruction methods to create 3D images.
\end{abstract}

\section{Introduction}

The objective of this work was the development of an imaging method, which enables the three-dimensional (3D) reconstruction of the internal structure of damaged materials and components with thermal NDT techniques. Thermographic imaging using an Infrared (IR)-camera for signal detection has a big advantage compared to ultrasonic imaging using acoustic transducers: it does not need any coupling media and a parallel detection of many camera pixels is possible (more than 1 million!). The main disadvantage of pulsed thermography is the degrading spatial resolution with increasing depth. This is the reason why often only 1D thermographic reconstruction is performed, which can be applied for layered structures, where the lateral extension is big compared to their depth.

\section{Method}

A new method in the field of thermographic imaging is the Virtual Wave Concept (VWC), which allows an employment of image reconstruction methods from ultrasonic imaging for thermographic signals [1]. Due to this new approach all known reconstruction methods of ultrasonic testing, e.g. time reversal and F-SAFT [2], are accessible immediately for thermographic imaging. Before using these imaging methods for a Thermo-Tomography, a virtual signal has to be calculated by applying a local transformation to the temporal temperature evolution measured on a sample surface. The following equation connects the temperature signal $T$ with the virtual wave signal $T_{\text {virt }}$ for the same position vector $\mathbf{r}$ but different time scales $t$ and $t^{\prime}$ :

$$
T(\mathbf{r}, t)=\int_{-\infty}^{\infty} T_{\text {virt }}\left(\mathbf{r}, t^{\prime}\right) K\left(t, t^{\prime}\right) d t^{\prime} \quad \text { with } \quad K\left(t, t^{\prime}\right) \equiv \frac{c}{\sqrt{\pi \alpha t}} \exp \left(-\frac{c^{2} t^{\prime 2}}{4 \alpha t}\right)
$$

Herein, $\alpha$ is the thermal diffusivity of the specimen and $c$ the virtual speed of sound. It can be discretized to produce a matrix equation $\mathbf{T}=\mathbf{K} \mathbf{T}_{\text {virt }}$, where $\mathbf{T}$ and $\mathbf{T}_{\text {virt }}$ are the vectors of the measured temperature signal and of the virtual wave signal. The equation can be inverted only with appropriate regularization, as the matrix $\mathbf{K}$ shows a deficiency in rank. Regularization methods are used to get the reconstructed virtual wave field from the measured thermographic signal. The introduced transformation can be used for every specimen shape in 2D or 3D.

\section{Results}

To apply the VWC even for the industry, fundamental enhancements were carried out. Eq. 1 is valid only for short pulses and internal heat sources. We have expanded the applications for the VWC also for testing opaque materials external optical-excitation. Similar to laser ultrasonics only half of the value of the virtual speed of sound is taken because the virtual wave has to travel from the surface to the internal structure, where it is reflected or scattered, and back to the surface again. As the kernel $\mathbf{K}$ is based on a linear system it is also possible to convolve this kernel with any temporal form of excitation. In Fig. 1 the principle of Thermo-Tomography using WWC is shown. The 3D thermographic reconstruction is produced from multiple projectional thermal images. The picture shows an experimental set-up for the 3D reconstruction of embedded steel spheres, which were heated up by electromagnetic induction. The IR camera measures the surface temperature on three different detector planes. In Fig. 2 the Thermo-Tomography is shown for the reconstruction of four embedded steel spheres. By acquiring the temperature data on more than one surface, the resolution and quality of thermographic reconstructions can be enhanced. 

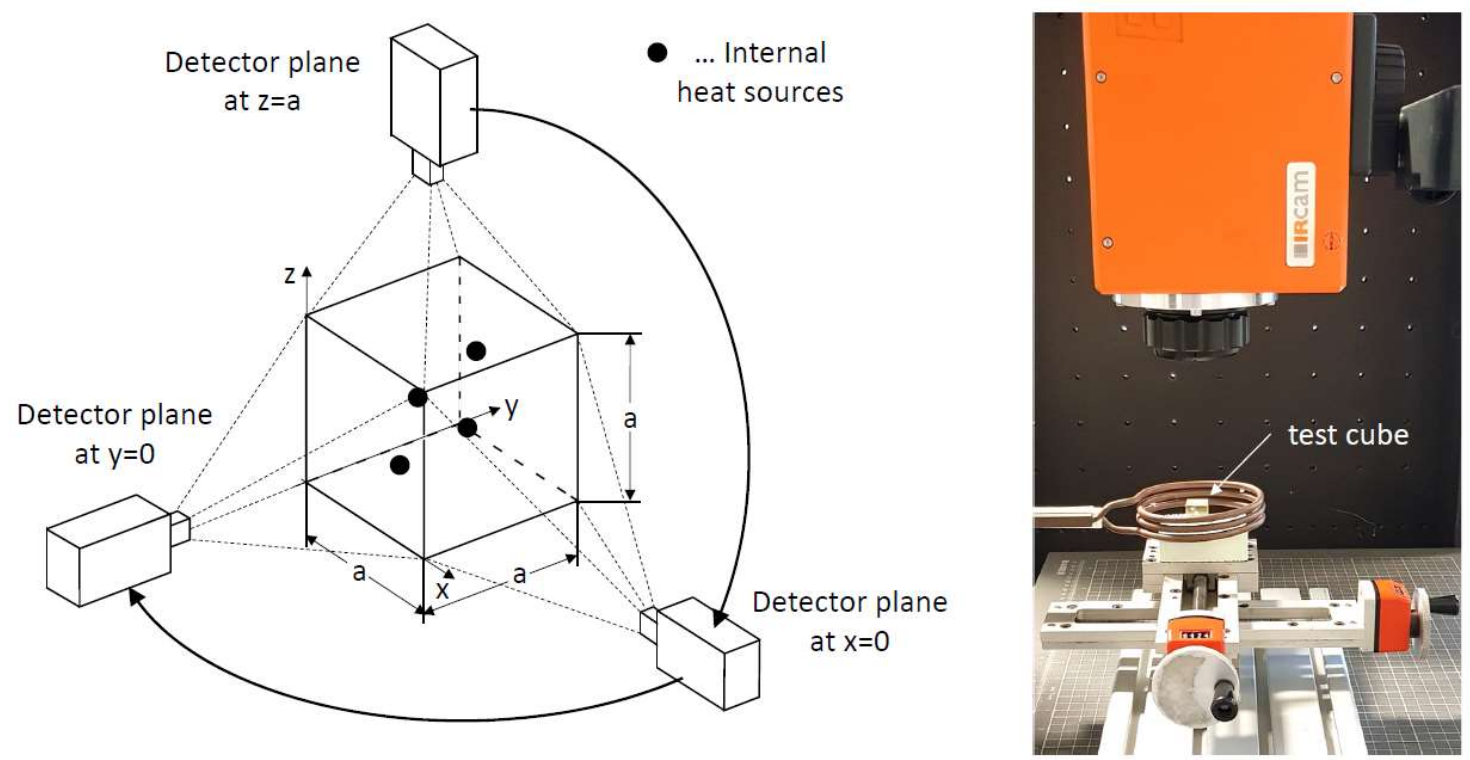

Fig. 1: Principle of Thermo-Tomography applying VWC: The 3D thermographic reconstruction is produced from multiple projectional thermal images. The photo shows an experimental set-up for the $3 D$ reconstruction of embedded steel spheres which were heated up by electromagnetic induction. The IR camera measures the surface temperature on the three different detector planes.
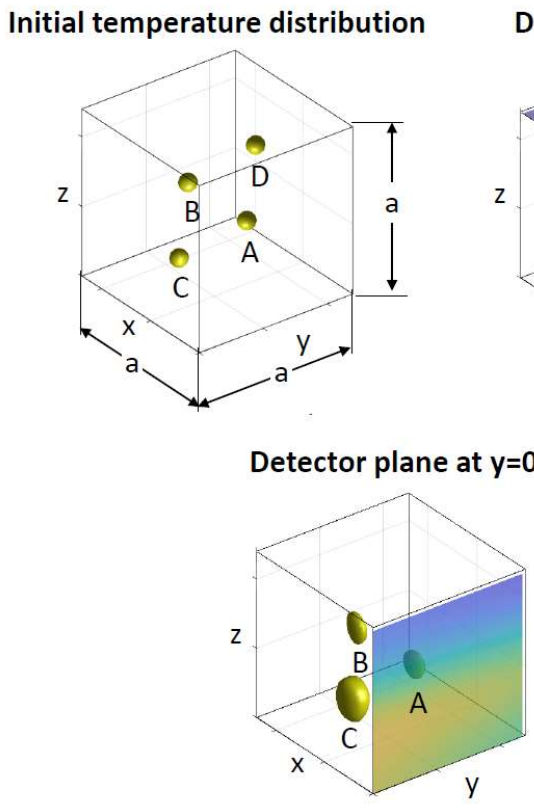
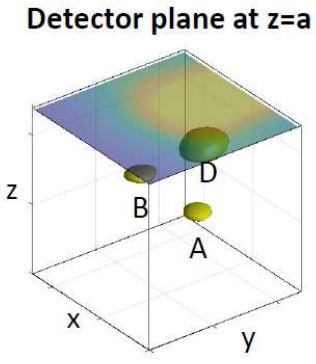

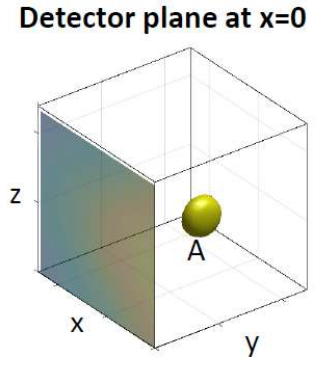

Fig. 2: Application of the VWC for the 3D reconstruction of the embedded steel spheres (see Fig.1). By acquiring the temperature data on more than one surface, the resolution and quality of thermographic reconstructions can be enhanced.

\section{Acknowledgment}

The financial support by the Austrian Federal Ministry of Science, Research and Economy and the National Foundation for Research, Technology and Development is gratefully acknowledged.

\section{REFERENCES}

[1] P. Burgholzer et al, J. Appl. Phys. 121, 10.1063/1.4978010 (2017).

[2] J. Krautkraemer and H. Krautkraemer, Ultrasonic Testing of Materials (Springer, 1990). 